\title{
Plage and flare Activity of the RS CVn-type Star UX Arietis during 2001-2002
}

\author{
Sheng-hong Gu $\dagger$ \\ National Astronomical Observatories/Yunnan Observatory, Chinese Academy of Sciences, \\ Kunming, China \\ e-mail: shenghonggu@ynao.ac.cn
}

\begin{abstract}
The very active RS CVn-type star UX Ari was observed using high-resolution echelle spectrograph attached to the $2.16 \mathrm{~m}$ telescope of Xinglong station in Nov.-Dec. 2001 and Dec. 2002. By means of synthetic spectral subtraction method, the information about chromospheric activity of the system was obtained through several chromospheric activity indicators $\mathrm{HeI} \mathrm{D}_{3}$, $\mathrm{NaI} \mathrm{D}_{1} \mathrm{D}_{2}, \mathrm{H}_{\alpha}$, and CaII IRT lines. Based on the analysis for these activity indicators, we found that the chromospheric activity of UX Ari showed obvious orbital modulation phenomenon, and the favorite active longitudes were around the quadratures of the binary system. During the two observing runs, hot plage and very strong optical flare events were detected, which were always happened around the favorite active longitudes of the system. Moreover, they were linked with the photospheric starspots in spatial structure, and appeared just above the main starspots.
\end{abstract}

Keywords. binaries: spectroscopic, stars: chromospheres, stars: activity, stars: flare

\section{Introduction}

UX Ari is one of the most active RS CVn-type stars, which shows significant photometric variability, long-term starspot activity (Aarum \& Henry 2003; Aarum et al. 1999; $\mathrm{Gu}$ et al. 2003; $\mathrm{Gu}$ et al. 2004); strong $\mathrm{H}_{\alpha}$ emission above continuum spectrum, CaII H\&K, IRT core emission, and optical flares (Aarum \& Engvold 2003; Gu et al. 2002; Montes et al. 1996, 2000). It is a triple-lined spectroscopic triple system with spectral type $\mathrm{G} 5 \mathrm{~V}+\mathrm{K} 0 \mathrm{IV}+\mathrm{K} 5 \mathrm{~V}$ and orbital period $6^{d} .4378553$ for the binary (primary and secondary) (Duemmler \& Aarum 2001), and its observational spectrum is very complicated. The previous research demonstrates that the chromospheric activity of this system is mainly associated with the cooler secondary component. In order to investigate the scale and evolution of the solar-like activity on UX Ari, we began a long-term monitoring project for it by means of high-resolution spectroscopy in 2000. Here we present the preliminary result about its chromospheric activity based on several chromospheric activity indicators $\mathrm{HeI} \mathrm{D}_{3}, \mathrm{NaI} \mathrm{D}_{1} \mathrm{D}_{2}, \mathrm{H}_{\alpha}$, and CaII IRT lines in 2001-2002.

\section{Observation and data reduction}

The new observations were made with Coude echelle spectrograph (Jiang 1996; Zhao \& Li 2001) of the $2.16 \mathrm{~m}$ telescope at Xinglong station of NAOC during two observing runs: Nov. 23-Dec. 12001 and Dec. 13-17 2002. The spectral resolution is about 36000. A $1 \mathrm{k} \times 1 \mathrm{k}$ Tektronix CCD detector was used in the observations. The reciprocal dispersions were $0.081 \AA /$ pixel for HeI and NaI, $0.091 \AA /$ pixel for $\mathrm{H}_{\alpha}$, and $0.120 \AA /$ pixel for CaII line spectral regions, respectively. The signal to noise ratio $(\mathrm{S} / \mathrm{N})$ was more than 100 for most of the observations. All observed spectral images were reduced by using IRAF

$\dagger$ Present address: P.O. Box 110, Kunming, Yunnan Province, China. 

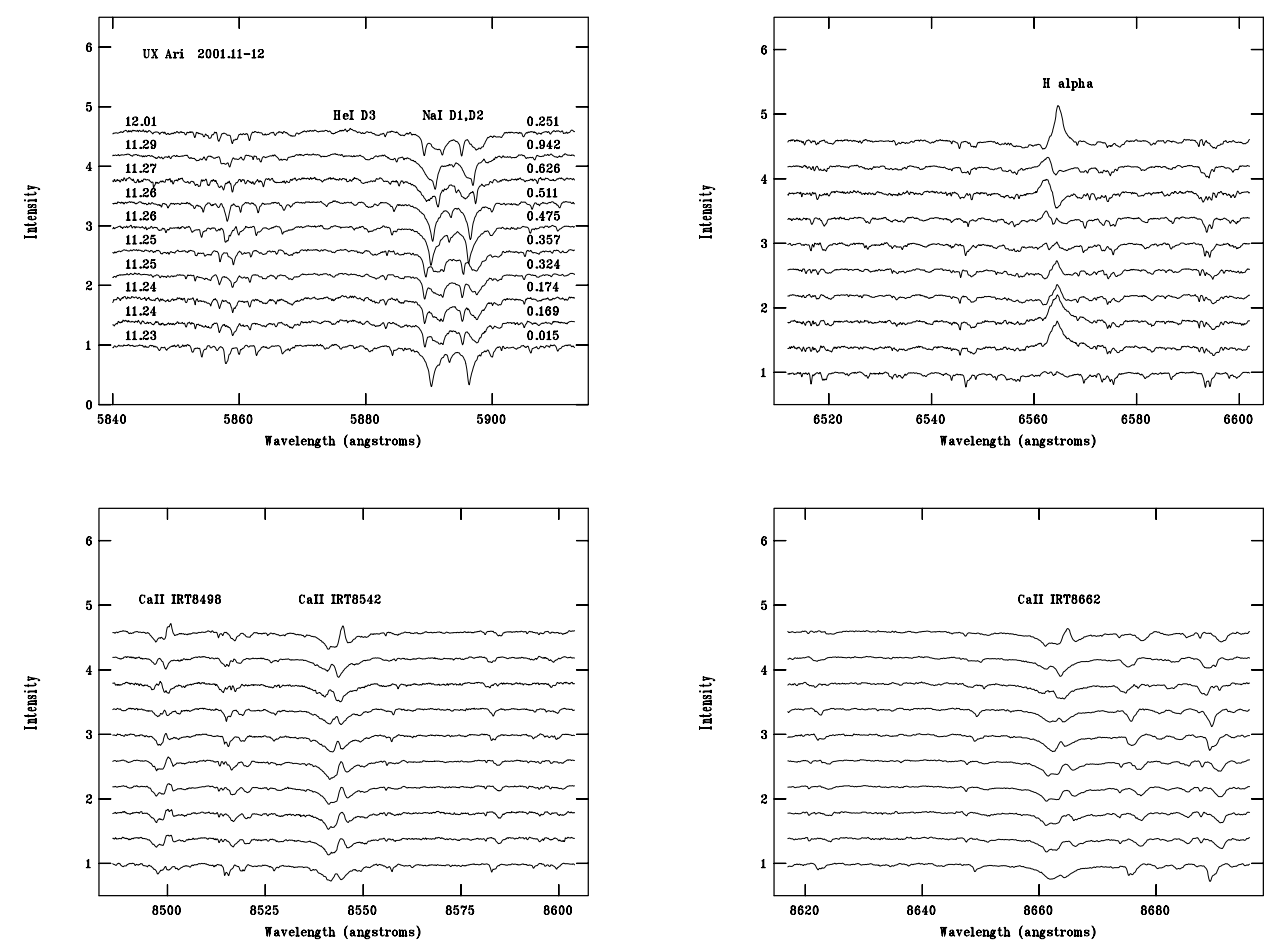

Figure 1. The chromospheric activity indicators in Nov.-Dec. 2001.

package according to the standard fashion. Several chromospheric activity indicators in our observations are displayed in Figure 1 and 2 .

\section{Spectral synthesis}

Because UX Ari is a triple system, the spectra of its components are mixed together in the observed spectra. Therefore, it is hard to measure chromospheric emission of the active component directly. In order to derive the pure chromospheric emission information, we use synthesized spectral subtraction technique (Barden 1985; Montes et al. 1995) to do data analysis. In this method, the synthesized spectrum for the system is constructed from artificially rotationally broadened, radial-velocity shifted, and weighted spectra of three inactive stars with the same (similar) spectral-type and luminosity class as three components of the system. Then the excess chromospheric emission can be derived by removing the synthesized spectrum from the observed one. In our observing runs, we observed three inactive template stars HR3309 (G5V), HR3351 (K0IV) and HR753 (K3V) for the primary, secondary and tertiary, respectively. The examples for spectral subtraction technique are shown in Figure 3.

\section{Orbital modulation, plage and flare}

From Figure 1 and 2, it can be found that the chromospheric activity indicators vary following orbital phase, and the behavior of each indicator is consistent to the others. This suggests that there is an orbital modulation phenomenon for the chromospheric activity of UX Ari. The measured equivalent width EWs of $\mathrm{H}_{\alpha}$ excess emission in our two 

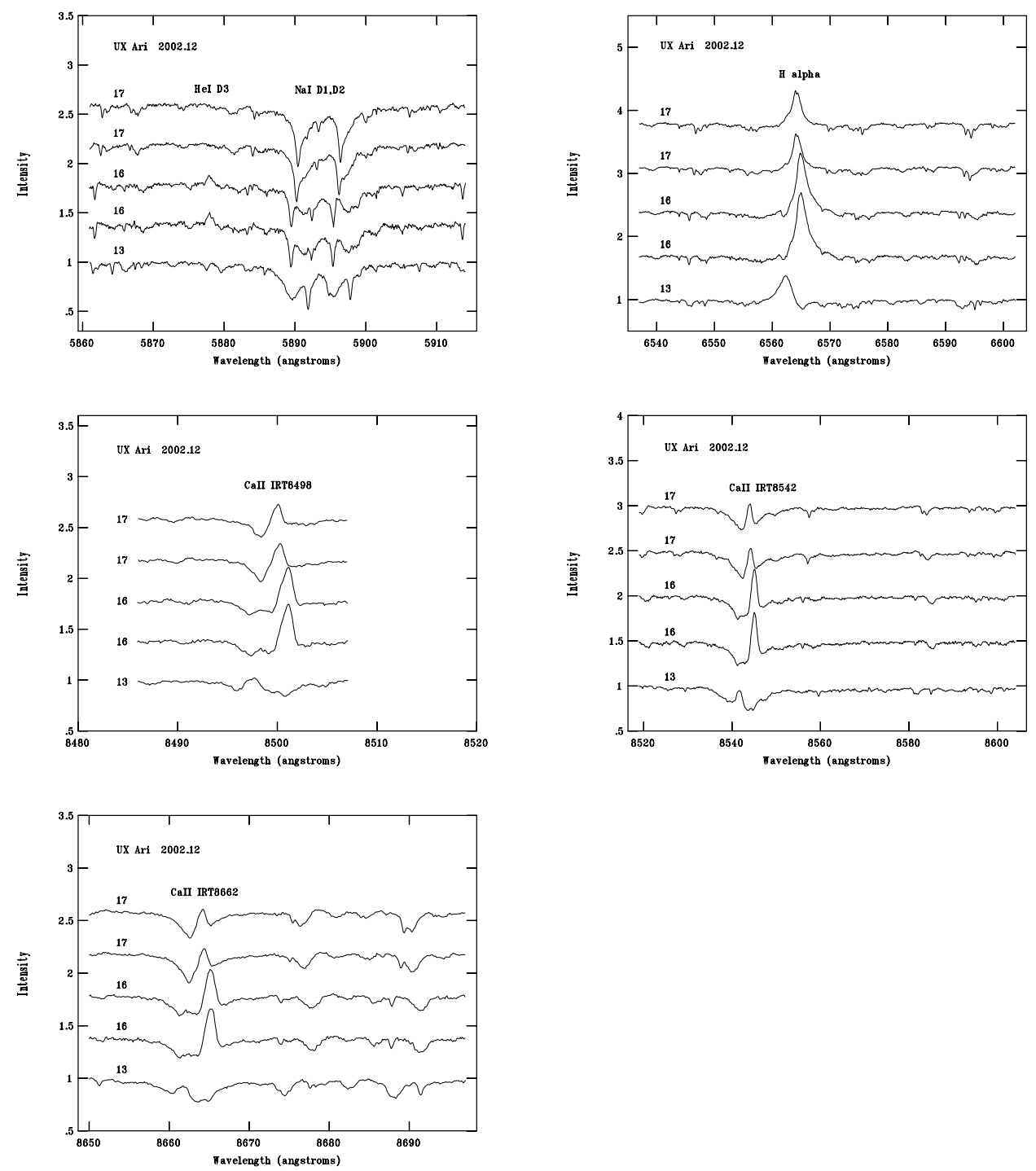

Figure 2. The chromospheric activity indicators in Dec. 2002.

observing runs have been plotted vs the orbital phase in Figure 4. In the first observing run, the spectra at orbital phases $0.169,0.174$, and 0.251 show stronger excess emission for all indicators. In these phases, the $\mathrm{HeI} \mathrm{D}_{3}$ line shows weaker emission, this means that we detected a hot plage-like event in that run (Berdyugina et al. 1999). In the second observing run, the spectra at orbital phases 0.280 and 0.292 exhibit very strong excess emission in all indicators. In these phases, the $\mathrm{H}_{\alpha}$ and CaII IRT emission lines show a dramatic increase, the HeI $\mathrm{D}_{3}$ line changes to emission, and the $\mathrm{NaI} \mathrm{D}_{1} \mathrm{D}_{2}$ lines show a stronger fill-in. This means that a flare-like event was detected in the second run. By means of the calibration of Hall (1996) as the relation of color index (V-R), we convert the excess emission $\mathrm{EW}$ of $\mathrm{H}_{\alpha}$ line into luminosity, and derive the energy released in the flare, $4.7 \times 10^{31} \mathrm{erg} / \mathrm{s}$. Compared with the energy release in previous flare detected 

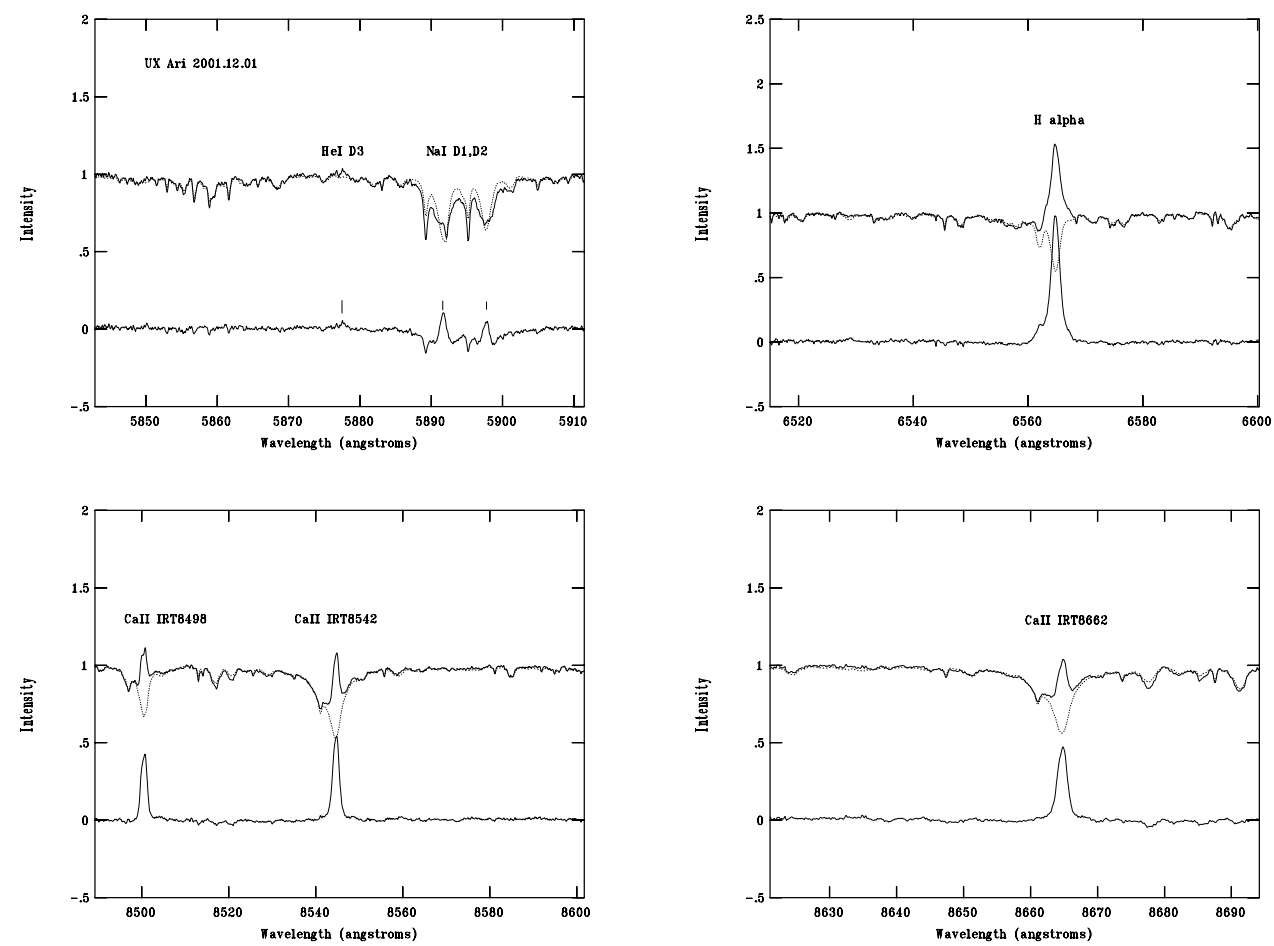

Figure 3. The examples for spectral subtraction technique.
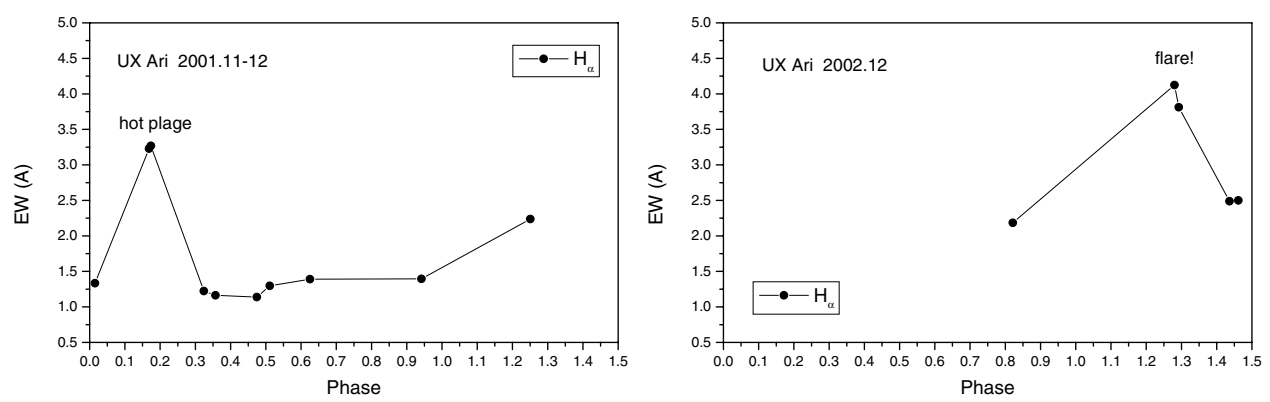

Figure 4. The EW of the excess emission vs orbital phase for $\mathrm{H}_{\alpha}$ line.

$\left(1.7 \times 10^{31} \mathrm{erg} / \mathrm{s}\right.$ (Montes et al. 1996$)$ and $2.1 \times 10^{31} \mathrm{erg} / \mathrm{s}(\mathrm{Gu}$ et al. 2002$\left.)\right)$, it can be found that this is the strongest flare detected for UX Ari in the optical wavelength.

\section{Correlation between chromospheric and photospheric activity}

In our first observing run, the orbital phase coverage is good so that we can reconstruct the starspot pattern of the photospheric surface ( $\mathrm{Gu}$ et al. 2004). This permits us to make a comparison between chromospheric and photospheric activity for UX Ari. In this run, the chromospheric active region is around phase 0.2. Based on the Doppler image of UX Ari ( $\mathrm{Gu}$ et al. 2004) in this observing run, two main starspot active regions locate around phase 0.25 . Thus, it is obvious that there is a spatial connection between the 
main starspots and the hot plage. This demonstrates that the hot plage is associated with the starspot active regions in spatial structure.

\section{Summary}

From our new observations and the previous observations for UX Ari, we can see that UX Ari is a very active system, and the plage-like and flare-like events are frequent. There is a obvious orbital modulation phenomenon for the chromospheric activity of the system, and the favorite active longitudes are around two quadratures of the system (i.e. orbital phase 0.25 and 0.75 ). The flare just appears around the position where the hot plage appeared before. The chromospheric active region is associated with the main starspots in spatial structure. In order to understand the behavior of the magnetic activity of UX Ari, it is necessary to do further more observations for the system.

\section{Acknowledgements}

I would like to thank Dr. Jian-yan Wei and Dr. Xiao-jun Jiang for supporting our research project in $2.16 \mathrm{~m}$ telescope of Xinglong station. This work is supported by NSFC under grant No. 10373023.

\section{References}

Aarum, V. et al. 1999, Proceedings of "Astrophysics with the Nordic Optical Telescope", Karttunen, H. \& Piirola, V. eds., University of Turku, 222

Aarum, V. \& Engvold, O. 2003, A\&SA 402, 1043

Aarum, V. \& Henry, G.W. 2003, A\&A 402, 1033

Barden, S.C. 1985, ApJ 295, 162

Berdyugina, S. et al. 1999, A\&A 349, 863

Duemmler, R. \& Aarum, V. 2001, A\&\&A 370, 974

$\mathrm{Gu}$, S.-h. et al. 2002, A\&\&A 388, 889

Gu, S.-h. et al. 2003, IAU Symp. 219, in press

Gu, S.-h. et al. 2004, Proceeding of 13th Cambridge Workshop "Cool Stars, Stellar Systems, and the Sun", in press

Hall, J.C. 1996, PASP 108, 313

Jiang, S.-y. 1996, in Ground-based Astronomy in Asia, ed. Kaifu, N., 335

Montes, D. et al. 1995, A\& A 294, 165

Montes, D. et al. 1996, A\&\& 310 , L29

Montes, D. et al. 2000, A\&AS 146, 103

Zhao, G. \& Li, H.-b. 2001, CJAA 1, 555

\section{Discussion}

Cheng Fang: What is the duration of the plage and sunspots? Did you find several periodical behavior of the emission of the plage?

Gu: 1) Because the time resolution is lower in our observation, we can not estimate the duration of the plages and starspots properly. 2) Due to the above reason, we also can not determine the periodical behavior of the plage.

DERE: Does this star show X-ray signatures of flare activity?

Gu: I don't notice the behavior of this star in X-ray wavelength, but for most RS-CVn stars, there is very strong X-ray emission when the flare event is occurring. So, I guess this star should show stronger X-ray emission during the flare event. 